\title{
Research Paper \\ Effect of a 6-Week Resistance Training Program on Transforming Growth Fac- tor Beta-1 and Myostatin Genes Expression in Tendons of Extensor Digitorum Longus and Soleus Muscles in Rats
}

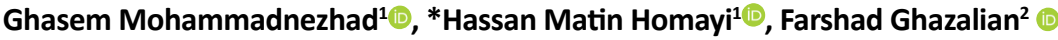

1. Department of Physiology, Faculty of Physical Education and Sports Science, Central Tehran Branch, Islamic Azad University, Tehran, Iran. 2. Department of Physiology, Science and Research Branch, Islamic Azad University, Tehran, Iran.

\begin{tabular}{|c|c|}
\hline $\begin{array}{l}\text { Use your device to scan } \\
\text { and read the article online }\end{array}$ & dtation: Mohammadnezhad Gh, Matin Homayi H, Ghazalian F. [Effect of a 6-Week Resistance Training Program on Trans- \\
\hline aptista & $\begin{array}{l}\text { forming Growth Factor Beta-1 and Myostatin Genes Expression in Tendons of Extensor Digitorum Longus and Soleus Mus- } \\
\text { cles in Rats (Persian)]. Journal of Arak University of Medical Sciences (JAMS). 2020; 23(1):82-91. https://doi.org/10.32598/ } \\
\text { JAMS.23.1.5849.1 }\end{array}$ \\
\hline 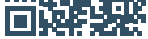 & dol' https://doi.org/10.32598/JAMS.23.1.5849.1 \\
\hline
\end{tabular}

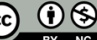

Article Info:

Received: 15 Dec 2019

Accepted: 21 Jan 2020

Available Online: 01 Apr 2020

Key words:

Resistance training

Tendon, TGF- $\beta 1$

Myostatin.

\section{A B S T R A C T}

Background and Aim Tendon is the extracellular matrix of the muscle that mechanically and structurally adapts to the mechanical load. However, the cellular and molecular mechanisms of this adaptation are not known yet. The purpose of this study was to investigate the effect of 6 weeks of resistance training on expression of two Transforming Growth Factor Beta 1 (TGF- $\beta 1$ ) and myostatin genes in the tendon of fast- and slow-twitch muscles including Extensor Digitorum Longus (EDL) and Soleus (SOL).

Methods \& Materials Twelve male Wistar rats with 8 weeks of age were randomly divided into two groups of exercise $(n=6)$ and control $(n=6)$. The exercise group performed resistance training (Carrying weights with $40-160 \%$ body weight on the ladder) for 6 weeks, 5 sessions per week. Forty-eight hours after the last training session, all rats were sacrificed and the tendons of SOL and EDL muscles were extracted. The mRNA expression level of TGF- $\beta 1$ and myostatin genes was assayed using real time polymerase chain reaction. Independent t-test was used for statistical analysis.

Ethical Considerations All experiments on animals were according to the ethical guidelines of Research Ethics Committee of Islamic Azad University, Central Tehran Branch (Ethical Code: IR.IAU.PS.REC.1398.296) Results The expression of TGF- $\beta 1$ gene in EDL $(P \leq 0.001)$ and $S O L(P \leq 0.01)$ muscle tendons significantly increased, while the expression of myostatin gene in EDL $(P \leq 0.001)$ and $S O L(P \leq 0.05)$ tendons were significantly reduced.

Conclusion Resistance training appears to up-regulate the basal levels of TGF- $\beta 1$ gene and down-regulate the basal levels of myostatin gene in tendons of fast- and slow-twitch muscles, where these effects are significantly more pronounced in the tendon of fast-twitch muscle.

\section{Extended Abstract}

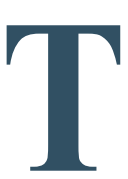

\section{Introduction}

Tendon is the Extracellular Matrix (ECM) of muscle that mechanically and structurally adapts to the mechanical load [2]. While the mechanical and morphological changes in response to resistance training in tendons are well documented, less is known about the basic cellular and molecular mechanisms that regulate these responses. It seems that the messaging pathway of the Transforming Growth Factor Beta 1 (TGF- $\beta 1$ ) plays a key role in adaptation of the tendon muscle to resistance training. Myostatin is a member of the

* Corresponding Author:

Hassan Matin Homayi, PhD.

Address: Department of Physiology, Faculty of Physical Education and Sports Science, Central Tehran Branch, Islamic Azad University, Tehran, Iran.

Tel: +98 (912) 3680810

E-mail: hasanmatinhomaee@gmail.com 
TGF- $\beta$ family, the expression of which negatively regulates skeletal muscle growth [5]. Both TGF- $\beta 1$ and myostatin stimulate tendon fibroblast proliferation and type I collagen synthesis [4, 6]. Based on laboratory studies on adult tendon fibroblasts and collagen synthesis studies in adult subjects in response to resistance training, it is thought that TGF- $\beta 1$ and myostatin play an important role in the growth and adaptation of adult tendons to resistance training [11]. The aim of this study was to investigate the effect of 6 weeks of resistance training on the expression of TGF- $\beta 1$ and myostatin genes in Tendons Of Soleus (SOL) and Extensor Digitorum Longus (EDL) muscles of Vistar male rats.

\section{Materials and Methods}

In this study, 12 adult male Wistar rats with 8 weeks of age were randomly divided into two groups: exercise $(n=6)$ and control $(n=6)$. The exercise group performed resistance training for 6 weeks (Table 1). Forty-eight hours after the last training session, all ranks were sacrificed. Then, the tendons of SOL and EDL muscles of their right foot were immediately and carefully extracted and stored at $-80^{\circ} \mathrm{C}$ for subsequent measurements. Expression levels of TGF- $\beta 1$ and myostatin mRNAs were assayed using RealTimePCR. Independent t-test was used for statistical analysis.

\section{Results}

The results showed a significant difference between the mRNA values of TGF- $\beta 1$ and myostatin genes in EDL and SOL tendons of exercise group compared to the control group. MRNA expression of TGF- $\beta 1$ gene in EDL $(0.48 \pm 0.14$ in exercise group vs. $0.14 \pm 0.04$ in control group, $\mathrm{P}<0.001)$ and $\mathrm{SOL}(0.32 \pm 0.08$ in exercise group vs. $0.17 \pm 0.14$ in control group, $\mathrm{P}<0.01)$ muscles increased significantly, while the myostatin gene expression level in EDL $(0.27 \pm 0.1$ in exercise group vs. $0.56 \pm 0.07$ in control group, $\mathrm{P}<0.001)$ and $\mathrm{SOL}(0.21 \pm 0.07$ in exercise group vs. $0.29 \pm 0.05$ in control group, $\mathrm{P}<0.05)$ muscles decreased significantly (Figures 1 and 2).

\section{Discussion}

The results of the present study showed that after 6 weeks of resistance training program, the mRNA expression of TGF- $\beta 1$ gene in both EDL and SOL muscles increased significantly. On the other hand, it significantly reduced mRNA expression of myostatin gene in both muscles. Heinemeier et al. (2007) reported an increase in mRNA levels of the TGF- $\beta 1$ gene and type I and III collagens in tendon and skeletal muscle following isometric, concentric and eccentric contractions by stimulating the sciatic nerve for 4 days [14]. Evidence suggests that TGF- $\beta 1$ has been a major mediator in the induction of collagen synthesis in fibroblasts by mechanical load [14], and a similar role has been suggested for this gen in tendons [15]. A 245\% increase in TGF- $\beta 1 \mathrm{mRNA}$ expression in the EDL muscle tendon compared to an $81 \%$ increase in the SOL muscle tendon reported in the present study is likely to indicate a higher involvement of fast-twitch muscles in resistance training which leads to greater adaptations in collagen tissue and higher tolerance of the force exerted by the fasttwitch muscle to the tendon.

There is ample evidence that the regulation of myostatin is a characteristic of the type of muscle fibers, and is strongly associated with the myosin heavy chain IIb isoform [18] and the high concentration of myostatin protein in the contractile muscle. Has been [19], and high concentrations of myostatin protein have been observed in the fast-twitch muscle compared to the slow-twitch muscles [19]. These reports could justify the results of the present study regarding a 53\% reduction in myostatin mRNA expression in the EDL muscle compared to a slight $28 \%$ reduction in its expression in the SOL muscle.

In overall, resistance training appears to positively regulate the baseline mRNA levels of TGF- $\beta 1$ gene and negatively regulate the baseline mRNA levels of myostatin gene in fast- and slow-twitch muscles; where its effect was significantly higher on the fast-twitch muscle than the slowtwitch muscle.

Table 1. The resistance training protocol

\begin{tabular}{ccccccc}
\hline Week & $\mathbf{1}$ & $\mathbf{2}$ & $\mathbf{3}$ & $\mathbf{4}$ & $\mathbf{6}$ \\
\hline Load (body weight percentage) & $40-50$ & $60-70$ & $80-90$ & $100-110$ & $130-140$ & $150-160$ \\
Sets & 5 & 5 & 5 & 6 & 8 \\
Repetitions & 8 & 8 & 8 & 8 \\
\hline
\end{tabular}


A) Tendon of EDL

Tendon of SOL

Training Control

Training Control

TGF- $\beta 1$

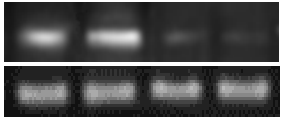

GAPDH

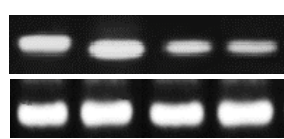

B)

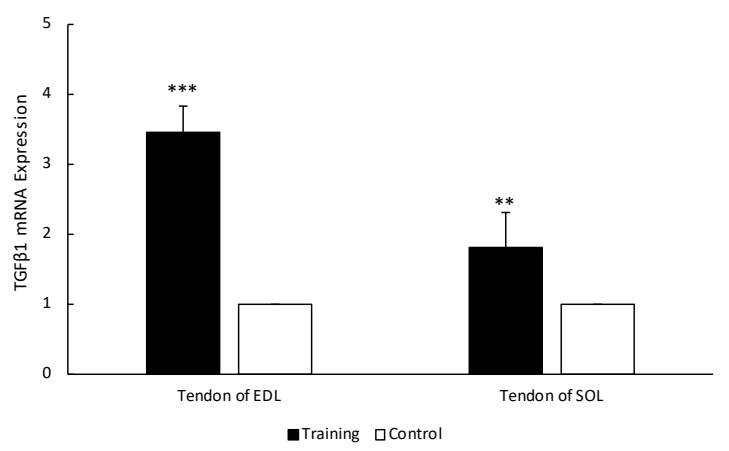

Journal of

rak University of Medical Sciences

Figure 1. a. RNA agarose gel electrophoresis and b. mRNA expression level of TGF- $\beta 1$ gene in the EDL and SOL muscles of rats in exercise and control groups. ${ }^{* *} \mathrm{P}<0.01 ; * * * \mathrm{P}<0.001$.

\section{Ethical Considerations}

\section{Compliance with ethical guidelines}

All experiments on animals were according to the ethical guidelines of Research Ethics Committee of Islamic Azad University, Central Tehran Branch (Ethical code: IR.IAU. PS.REC.1398.296)

\section{Funding}

The present paper was extracted from the $\mathrm{PhD}$ thesis of the first author, Ghasem Mohammadnezhad, Department of Physiology, Faculty of Physical Education and Sports Science, Central Tehran Branch, Islamic Azad University.

\section{Authors' contributions}

Conceptualization: All authors; Methodology and Data Analysis: Ghasem Mohammadnejhad; Editing and Review: all authors.

\section{Conflicts of interest}

The authors declare no conflict of interest.

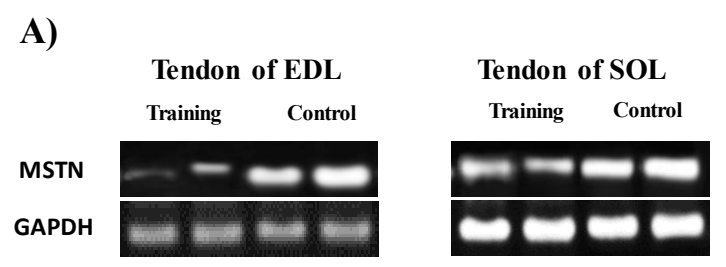

B)

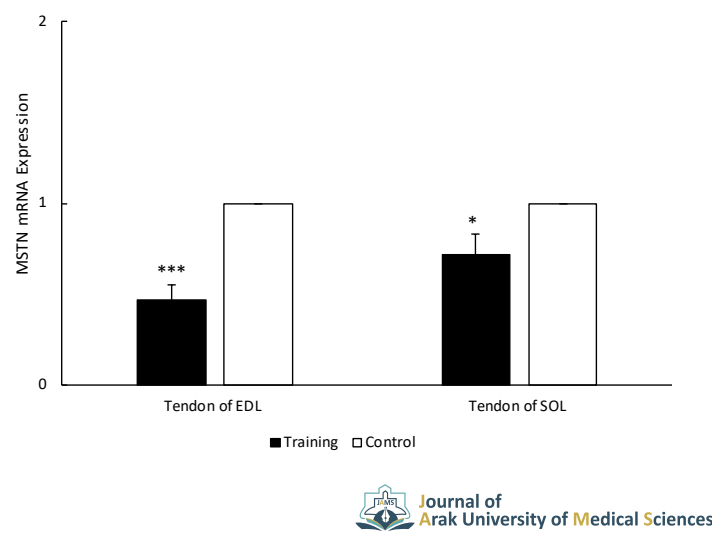

Figure 2. a. RNA agarose gel electrophoresis; and b. mRNA expression level of myostatin gene in the EDL and SOL muscles of rats in exercise and control groups. ${ }^{* *} \mathrm{P}<0.01$; $* * * \mathrm{P}<0.001$

\section{Acknowledgements}

The authors would like to thank the Department of Physiology and Pharmacology of the Pasteur Institute of Iran, as well as the head of the Laboratory Animal Housing of Tarbiat Modares University for their cooperation in conducting this study. 


\title{
اثر و هفته تمرين مقاومتى بر بيان ثنهاى TGF-B1 و ميوستاتين در ثاندون عضالات EDL و سولئوس موشهاى تمرين مقاومتى ويستار
}

\author{
قاسم محمدنزاده "حسن متين همائى ثه، فرشاد غزاليانه \\ ا. كروه فيزيولوزى ورزشى، دانشكده تربيت بدنى و علوم ورزشى، واحد تهران مركزي، دانشعاه آزاد اسلامى، تهران، يراني

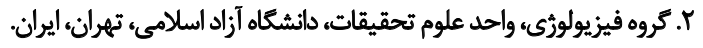

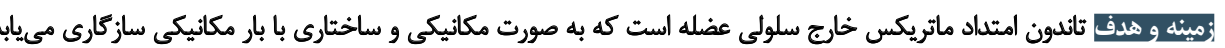

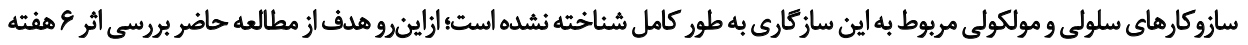

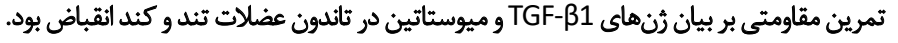

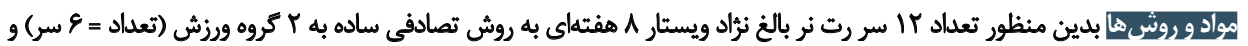

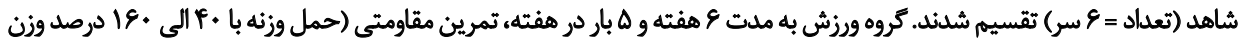

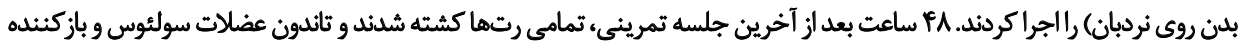

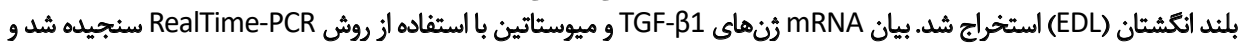

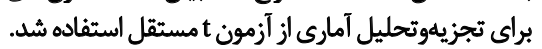

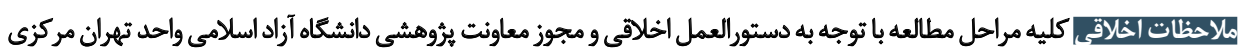

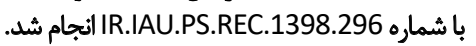

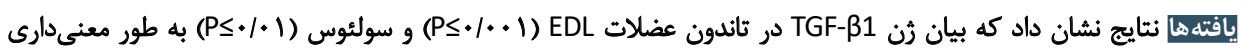

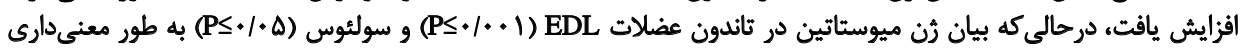
كاهش يافتي.

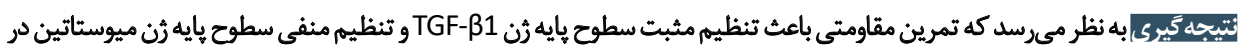

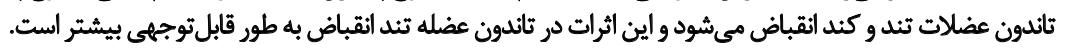

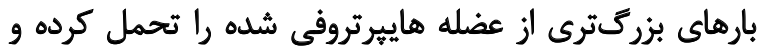

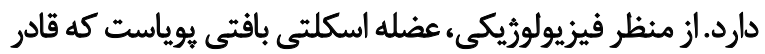

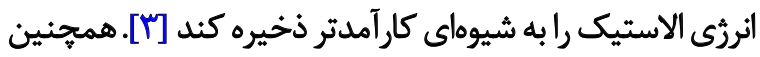

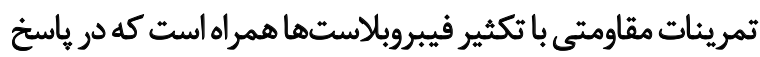

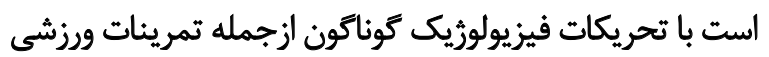

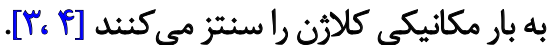
سازگًارى يابد [1]

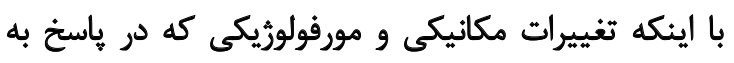

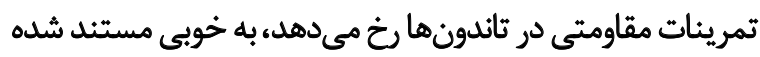

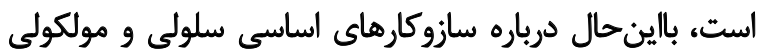

1. Cross-sectional area

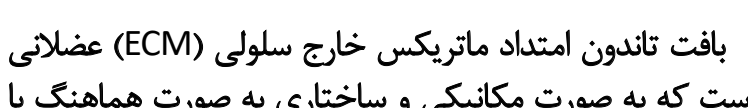

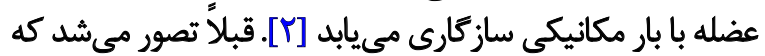

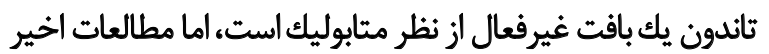

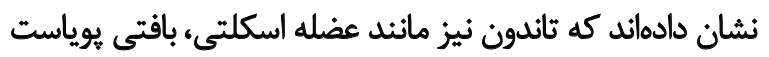

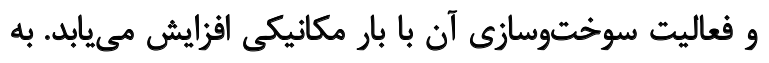

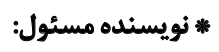

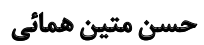

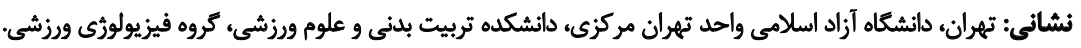
تلفن: + +

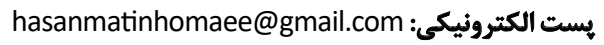


جدول ا. يروتكل تمرين مقاومتى

\begin{tabular}{|c|c|c|c|c|c|c|}
\hline ششم & هينجم & جهارم & سوم & دوم & اول & هفته \\
\hline $18 \cdot-10$. & $\mid f+-1 r+$ & $11 \cdot-1 .$. & $9 \cdot-\lambda$. & $\gamma+-\varepsilon$. & $\Delta \cdot-f_{*}$ & بار (درصد وزن بدن) \\
\hline 8 & 8 & 8 & $\Delta$ & $\Delta$ & a & تعداد ست \\
\hline$\Lambda$ & $\Lambda$ & $\Lambda$ & $\Lambda$ & $\Lambda$ & $\Lambda$ & تكرار در هر ست \\
\hline
\end{tabular}

آشناسازى با محيط نتخهدارى، رتها به روش تصادفى ساده به

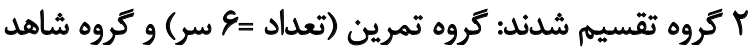

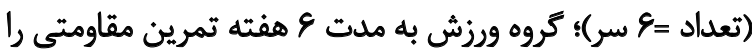

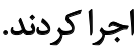
رتهاى كروه تمرين مقاومتى به منظور آشناسازى با تمرين به

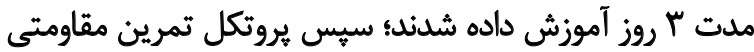

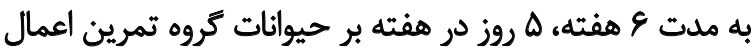

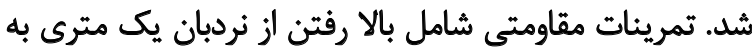

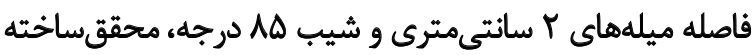

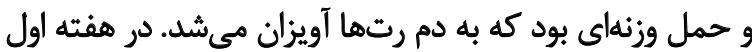

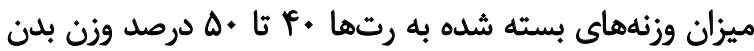

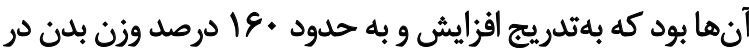

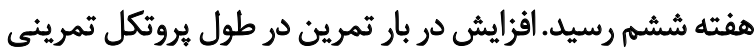

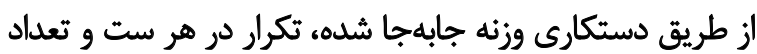

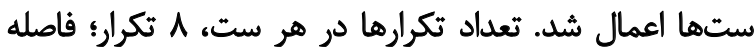

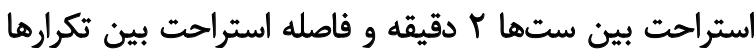

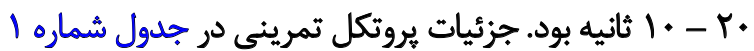

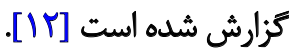

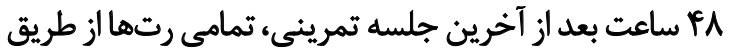

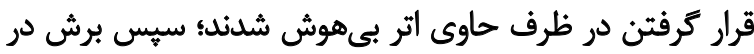

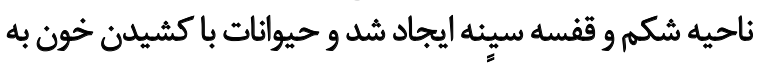

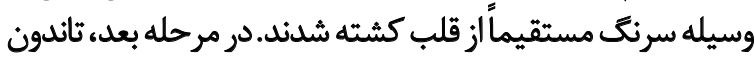

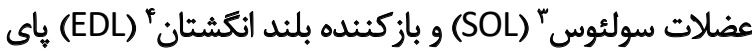

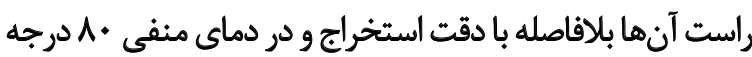
سانتى گراد براى سنجش هاى بعدى نكَهدارى شد.

به منظور استخراج RNA تام از بافت تاندون همورنشده، 1

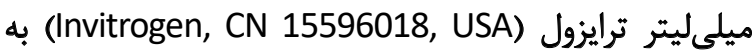

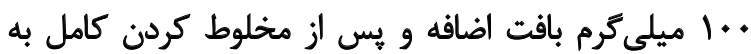

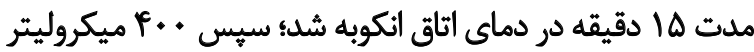
كلروفرم سرد (Merek, CAS 67-66-3 102445, Germany)

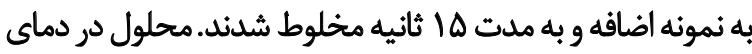

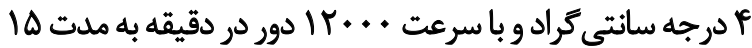

3. Soleus (SOL)

4. Extensor digitorum longus (EDL)
كه اين ياسخها را تنظيم مى كنيند، اطلاعات زيادى در دسترس

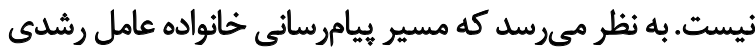

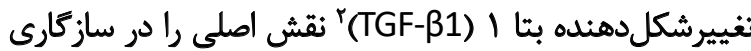

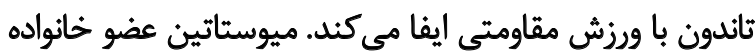

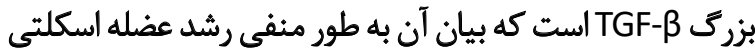

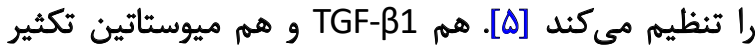

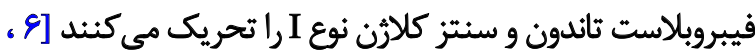

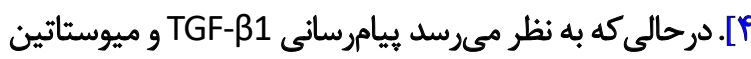

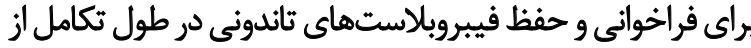

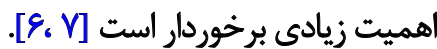

مطالعات متعددى وجود دارند كه توانايى اين سايتوكاينها

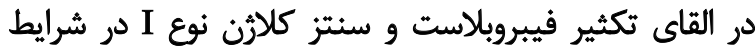

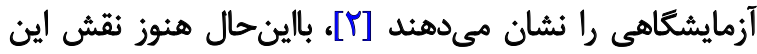

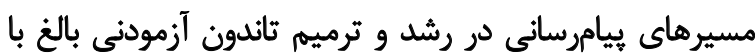

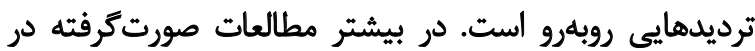

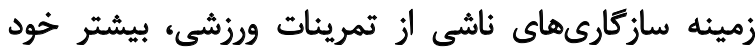

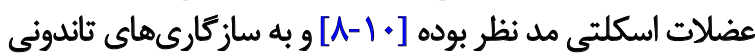

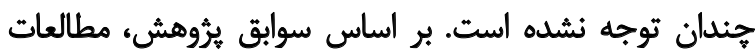

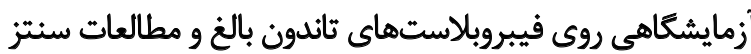

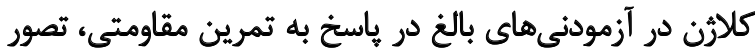

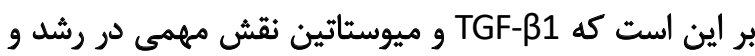

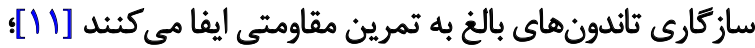

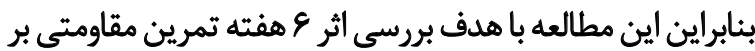

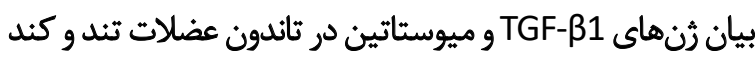
انقباض موشهاي نر ويستار انجام شد.

روش تحقيق

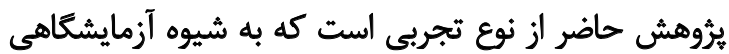

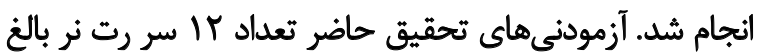

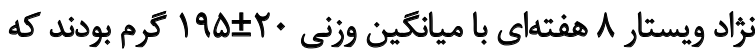

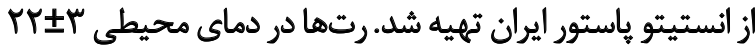

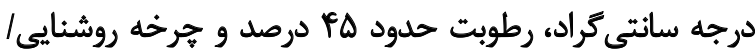

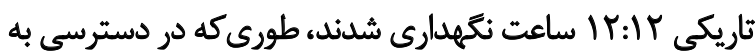

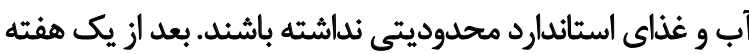

2. Transforming growth factor- $\beta 1$ (TGF- $\beta 1$ ) 
جدول r. توالى ئرايمرهاى مورد مطالعه در تحقيق حاضر.

\begin{tabular}{|c|c|c|c|c|c|}
\hline Accession Number & $\begin{array}{l}\text { Product } \\
\text { Length }\end{array}$ & Tm & Primer $\left(\phi^{\prime}\right.$ & $\begin{array}{c}\text { Forward/ } \\
\text { Reverse }\end{array}$ & Gene \\
\hline \multirow{2}{*}{$N M_{-} \cdot Y_{1 \Delta V \Lambda, r}$} & \multirow{2}{*}{$r \cdot}$. & $\Delta \Delta, I V$ & CAACAACGCAATCTATGACAA & FW & \multirow{2}{*}{ TGF- $\beta$ I } \\
\hline & & $\Delta f, \Delta r$ & CAAGGTAACGCCAGGAAT & REV & \\
\hline \multirow{2}{*}{ NM_.19101/1 } & \multirow{2}{*}{ VA } & $\Delta H^{\prime \prime}, 8 \Delta$ & CTACCACGGAAACAATCATTA & FW & \multirow{2}{*}{ Myostatin } \\
\hline & & $\Delta q, \cdot r$ & AGCAACATTTGGGCTTTCCAT & REV & \\
\hline \multirow{2}{*}{ 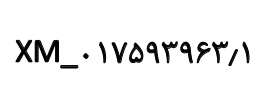 } & \multirow{2}{*}{$\| r$} & 81,01 & AAGTTCAACGGCACAGTCAAGG & FW & \multirow{2}{*}{ GAPDH } \\
\hline & & EI,H & CATACTCAGCACCAGCATCACC & REV & \\
\hline
\end{tabular}

براى اندازمخيرى سطوح بيان mRNA از روش كمّى Real

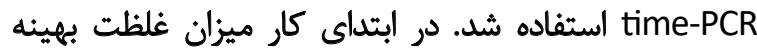

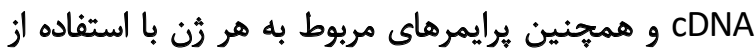

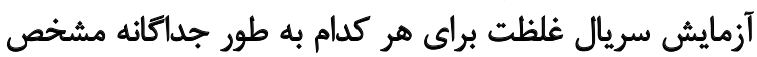

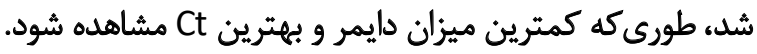

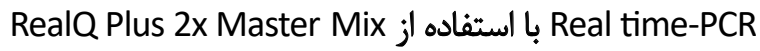

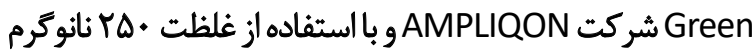

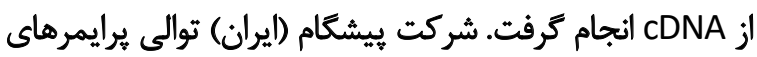

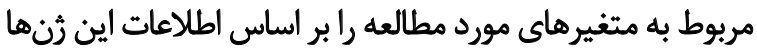

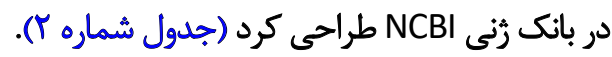

برنامه Real time-PCR شامل واسرشت اوليه در دماي هو

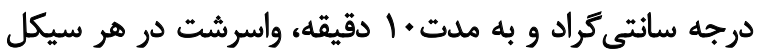

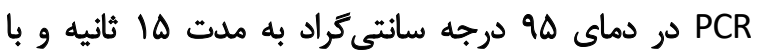

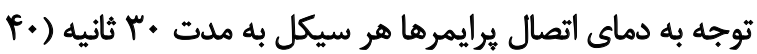

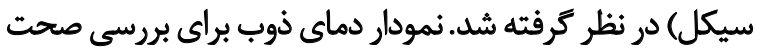

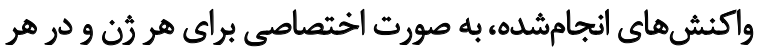

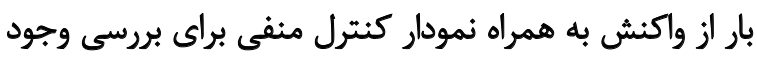

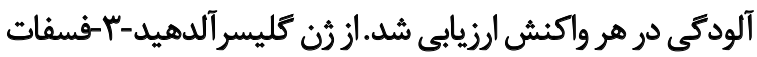

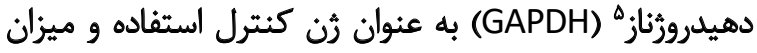

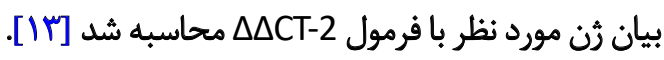

براى بررسى طبيعى بودن توزيع دادهها از آزمون كولموكروف-

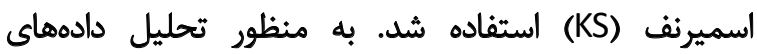

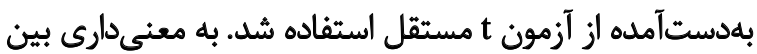

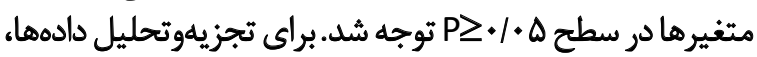
از نرمافزار Excel

Latiols

نتايج نشان داد كه بين مقادير MRNA رنهاي TGF-B1

5. Glyceraldehyde-3-phosphate dehydrogenase
دقيقه سانتريفيور شد. مايع حاوى RNA به ميكروتيوب ديكرى

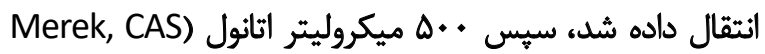

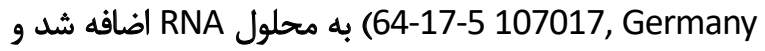

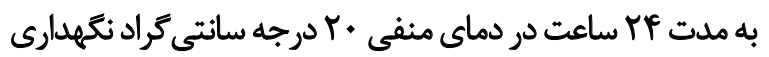

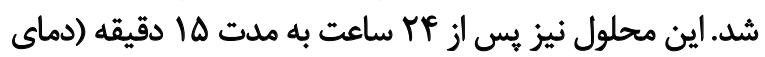

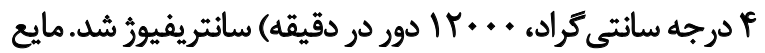

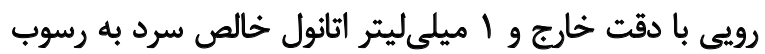

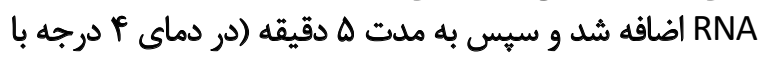

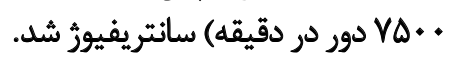

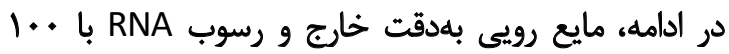

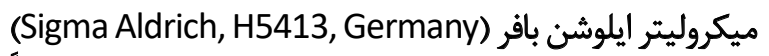
رقيق شد. تمام مراحل استخراج زير هود و با مواد و وسايل كاملاً

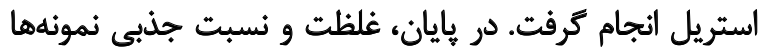
با استفاده از دستكاه اسيكتوفتومتر (Eppendorf, Germany)

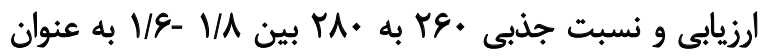

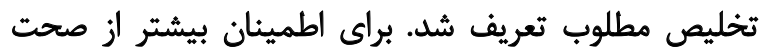

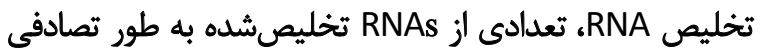

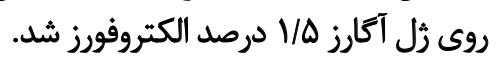

يس از استخراج RNA با خلوص و غُلظت بالا، مراحل سنتز

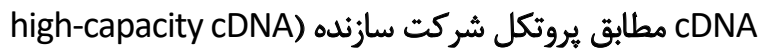
(reverse transcription kit

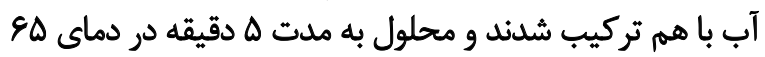

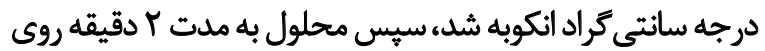

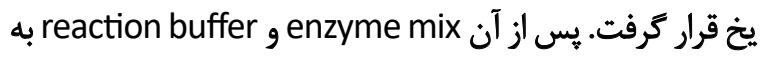

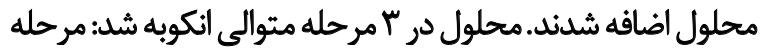

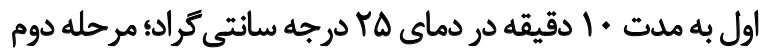

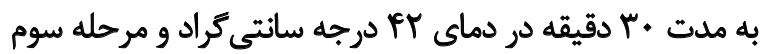

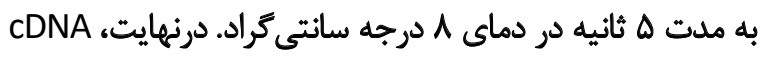

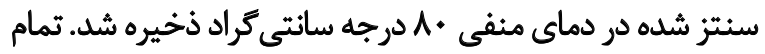

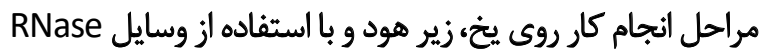
free انجام شد. أندام كول 
بررسى متون حاكى از آن است كه بيشتر مطالعات صورت كرفته

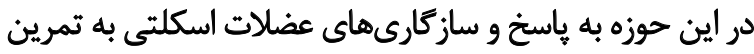

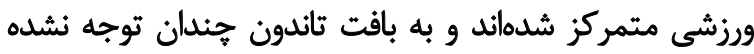

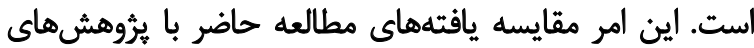

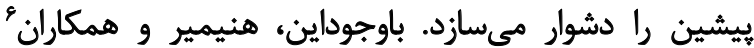

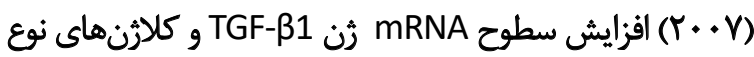

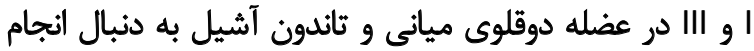

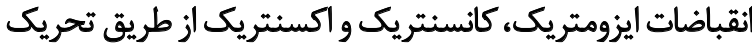

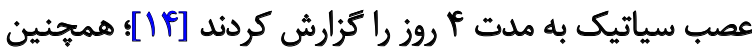
در تحقيق ديكرى نشان داده شد كه انجام تمرين استقامتى

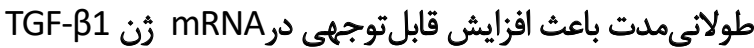

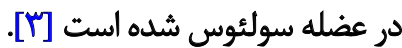

سازوكارهايى كه در طول تمرينات ورزشى بار مكانيكى را به

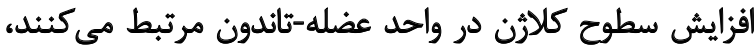

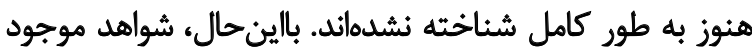

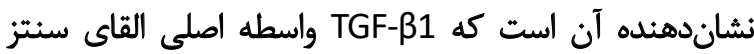

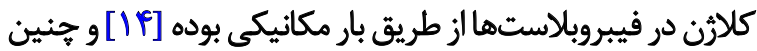

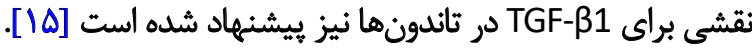

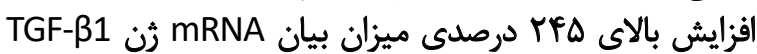

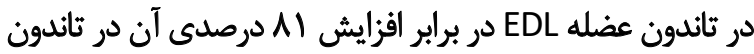

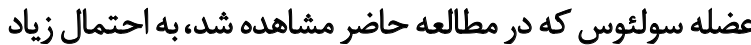

و ميوستاتين تأندون عضلات EDL و سولئوس كروه تمرين

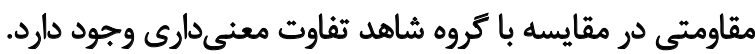

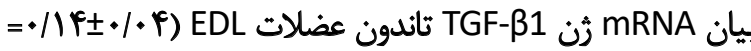

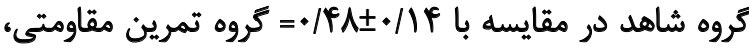

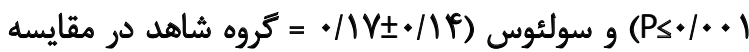

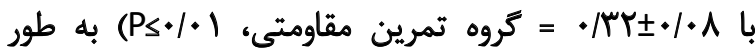

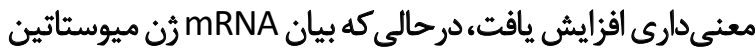
تاندون عضلات EDL

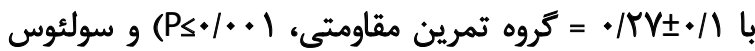

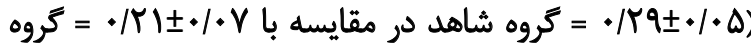

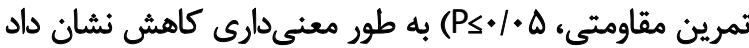

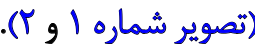

بحث

مطالعه حاضر با هدف بررسى اثر \& هفته تمرين مقاومتى بر

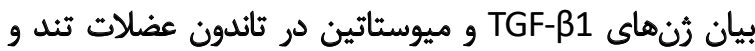

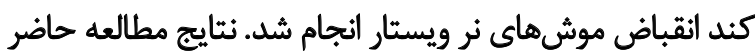

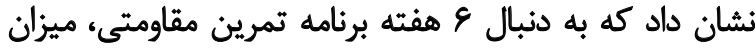

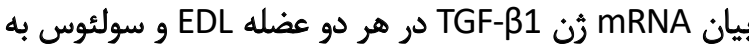

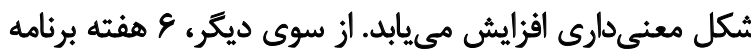

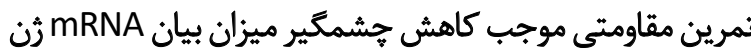

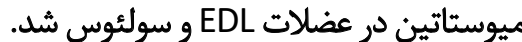

6. Heinemeier et al.

\begin{tabular}{|c|c|c|}
\hline & EDL تاندون عضله & SOL تاندون عضله \\
\hline & تروه تمرين & تروه شاهد كروه تمرين \\
\hline TGF- $\beta 1$ & -2 & $=$ \\
\hline GAPDH & cin & \\
\hline
\end{tabular}

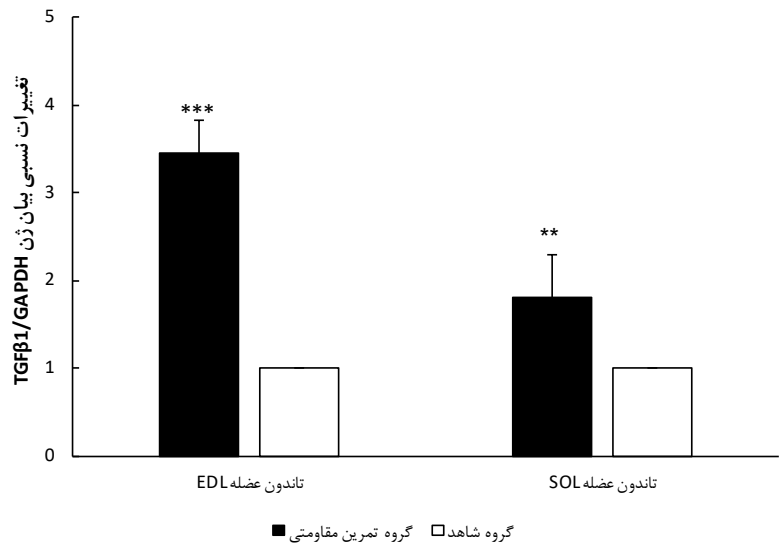

الف)

نمودار ا. الف) نمونه صحت تخليص RNA توسط الكتروفورز زل آكارز. ب) ميزان بيان ثن TGF-B در تاندون عضلات EDL و SOL

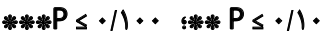



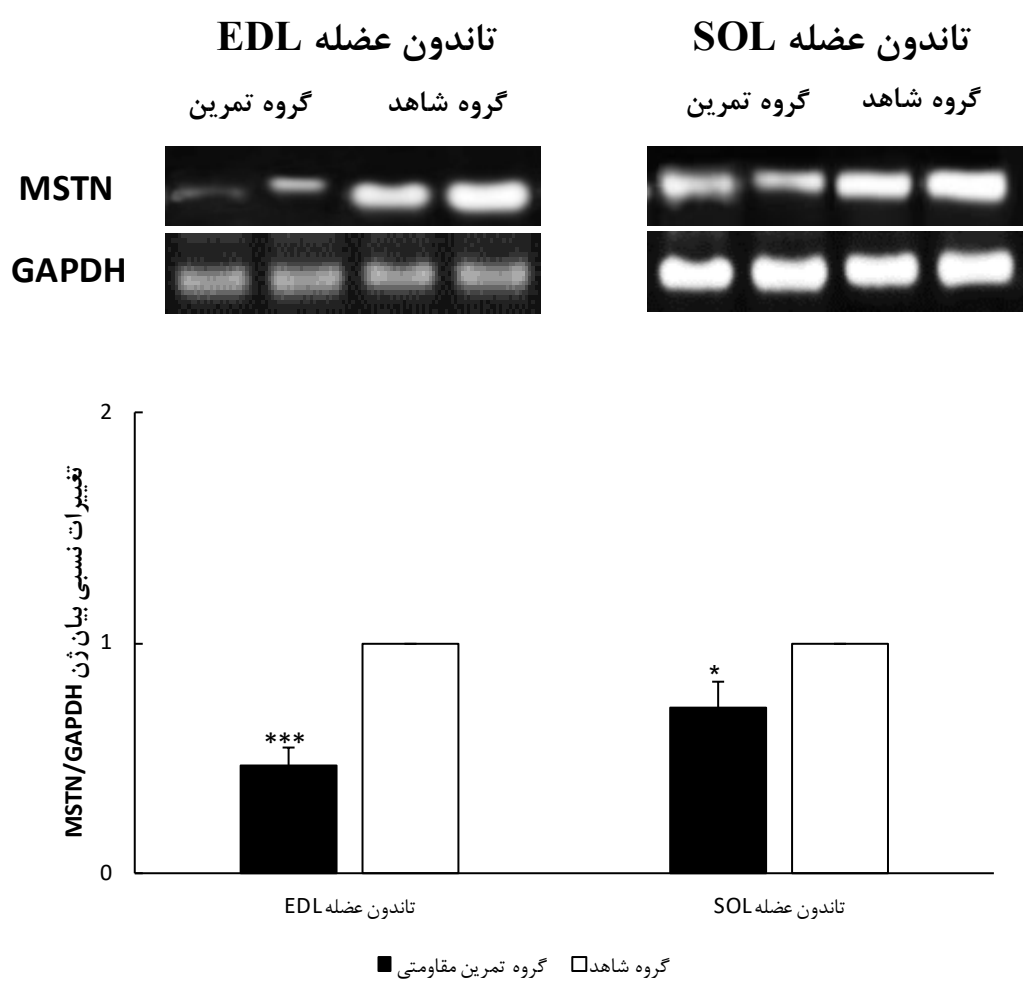

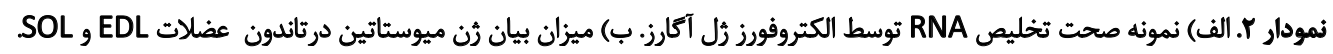
$* * * \mathrm{P} \leq / 11 \ldots * \mathrm{P} \leq \cdot / \mathrm{Q}$.

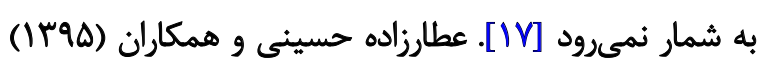

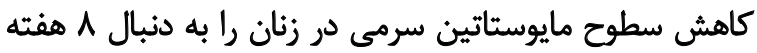

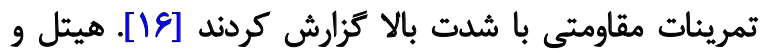

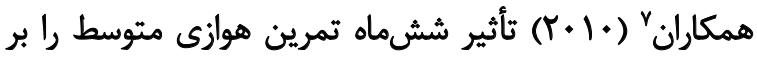

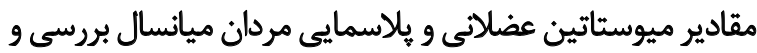

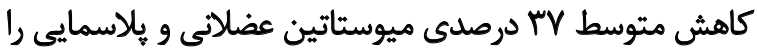

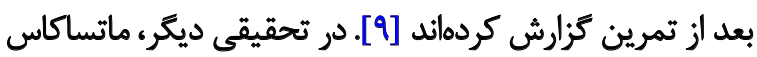

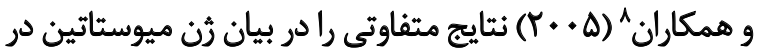

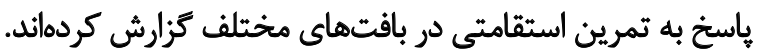

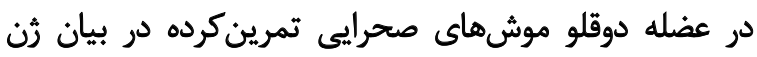

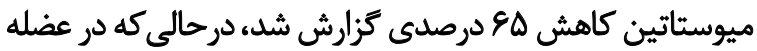

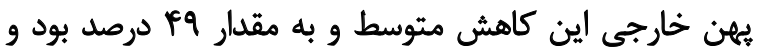

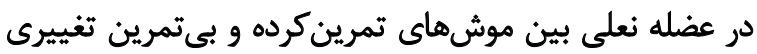

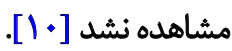

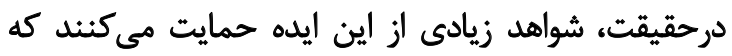

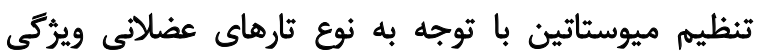

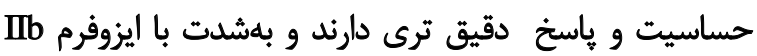

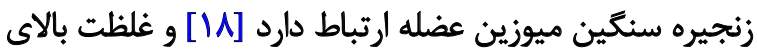

7. Hittel et al.

8. Matsakas et al.

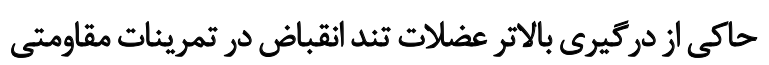

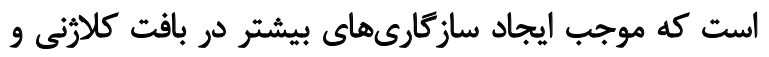
تحمل نيروى بالاتر وارده از سوى عضله تماري بند انقباض به به تاندون

$$
\text { هي تشود. }
$$

در مطالعه حاضر، تمرينات مقاومتى باعث كاهش سطوح بايه

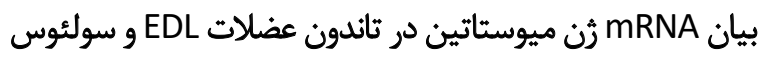
شد.كاهش سطوح سرمى و عضلانى ميوستاتين به دنبال تمرينات

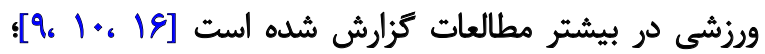

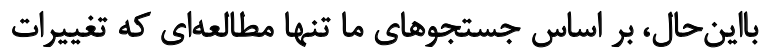

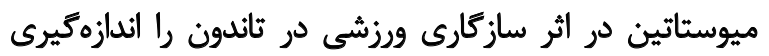

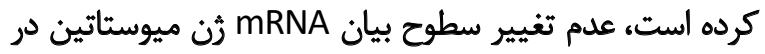

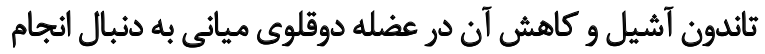

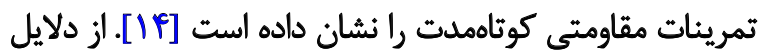

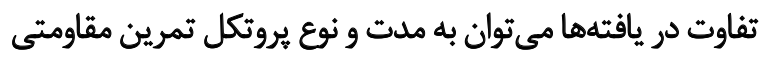

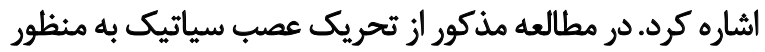

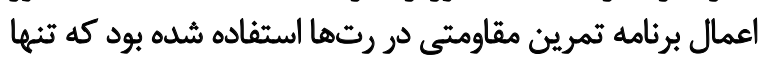

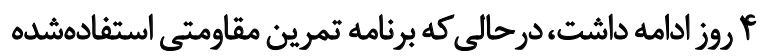

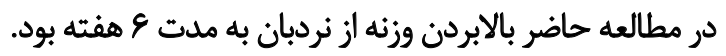
در تحقيقى جالب كزارش شده است كه نوع ورزش عامل

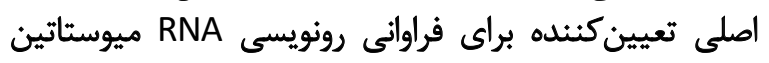




\section{مشار كث ثويسندكًان}

تعريـف موضـوع و بيـان مســأله: تمــام نويسـندكان؛ روش

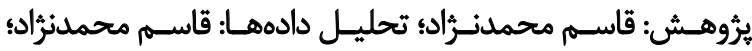

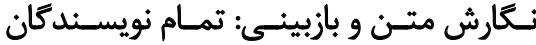

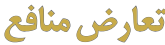

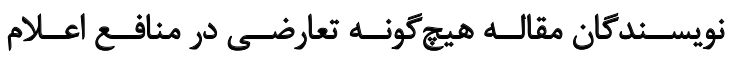

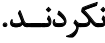

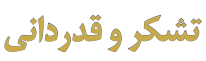

از برسنل محترم بخش فيزيولوزى و فارماكولوزى انستيتو

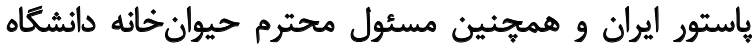

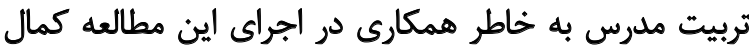
تشكر و قدردانى را داريم. ت باطي.
بروتئين ميوستاتين در عضلة تندانقباض در مقايسه با تارهاى

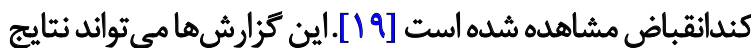

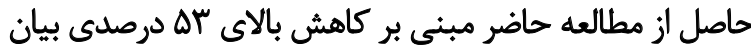

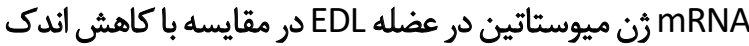
M Y درصدى بيان آن در عضله سولئوس را توجيه كند.

ميوستاثين علاوه بر اينكه اندازه، نوع و انقباضيذيرى عضلد

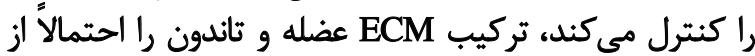

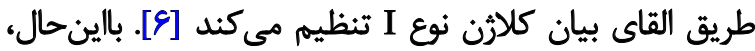

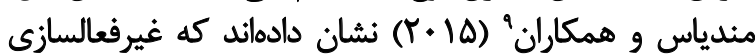

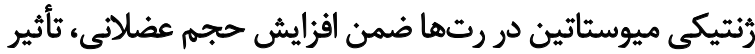

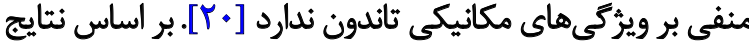

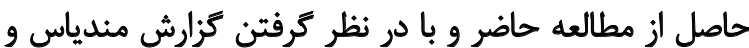

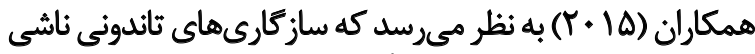

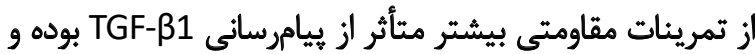

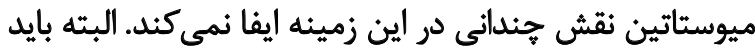

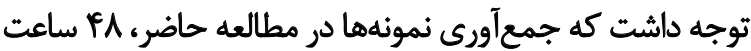

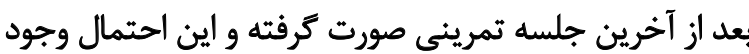

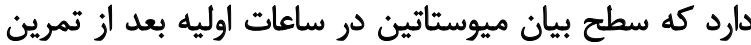

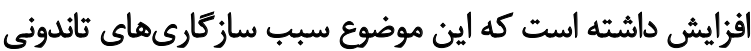

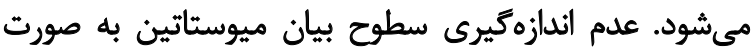

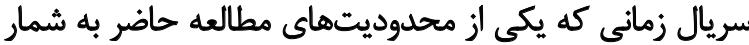
مىرود، اين تفسير را دشوار مى كند.

نتيجهيكيرى

بهاطوركلى، تمرين مقاومتى باعث تنظيم مثبت سطوح هايه

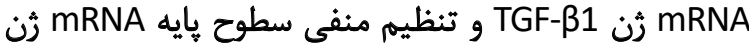

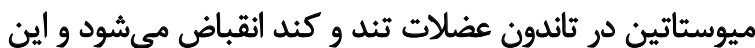

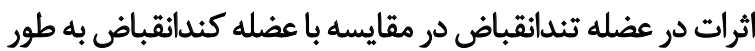
قابل توجهى بيشتر است.

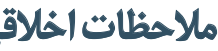

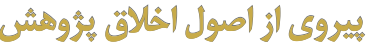

همه مر احل مربوط به حيوانات با توجه به دستور العمل اخلاقى

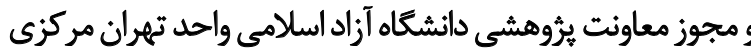
با شماره IR.IAU.PS.REC.1398.296 انجام شد.

$$
\text { حامى مالى }
$$

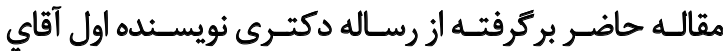

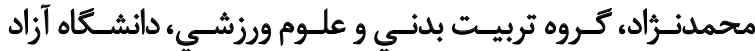

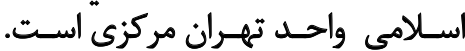

9. Mendias et al. 


\section{References}

[1] Schiaffino S, Reggiani C. Molecular diversity of myofibrillar proteins: Gene regulation and functional significance. Physiol Rev. 1996; 76(2):371-423. [DOI:10.1152/physrev.1996.76.2.371] [PMID]

[2] Davis ME, Gumucio JP, Sugg KB, Bedi A, Mendias CL. MMP inhibition as a potential method to augment the healing of skeletal muscle and tendon extracellular matrix. J Appl Physiol. 2013; 115(6):884-91. [DOI:10.1152/ japplphysiol.00137.2013] [PMID] [PMCID]

[3] Kjær M, Langberg H, Heinemeier K, Bayer M, Hansen M, Holm L, et al. From mechanical loading to collagen synthesis, structural changes and function in human tendon. Scandinavian J Med Sci Sports. 2009; 19(4):500-10. [DOI:10.1111/j.1600-0838.2009.00986.x] [PMID]

[4] Mendias CL, Gumucio JP, Bakhurin KI, Lynch EB, Brooks SV. Physiological loading of tendons induces scleraxis expression in epitenon fibroblasts. J Orthop Res. 2012; 30(4):606-12. [DOI:10.1002/jor.21550] [PMID] [PMCID]

[5] McPherron AC, Lawler AM, Lee SJ. Regulation of skeletal muscle mass in mice by a new TGF-p superfamily member. Nature. 1997; 387(6628):83 [DOI:10.1038/387083a0] [PMID]

[6] Mendias CL, Bakhurin KI, Faulkner JA. Tendons of myostatin-deficient mice are small, brittle, and hypocellular. Proc Natl Acad Sci. 2008, 105(1):388-93. [DOI:10.1073/pnas.0707069105] [PMID] [PMCID]

[7] Pryce BA, Watson SS, Murchison ND, Staverosky JA, Dünker N, Schweitzer R. Recruitment and maintenance of tendon progenitors by TGF $\beta$ signaling are essential for tendon formation. Development. 2009; 136(8):1351-61. [DOI:10.1242/dev.027342] [PMID] [PMCID]

[8] Czarkowska-Paczek B, Zendzian-Piotrowska M, Bartlomiejczyk I, Przybylski J, Gorski J. The effect of acute and prolonged endurance exercise on transforming growth factor-beta 1 generation in rat skeletal and heart muscle. J Physiol Pharmacol. 2009; 60(4):157-62. [PMID]

[9] Hittel DS, Axelson M, Sarna N, Shearer J, Huffman KM, Kraus WE. Myostatin decreases with aerobic exercise and associates with insulin resistance. Med Sci Sports Exerc. 2010; 42(11):2023-9. [DOI:10.1249/ MSS.0b013e3181e0b9a8] [PMID] [PMCID]

[10] Matsakas A, Friedel A, Hertrampf T, Diel P. Short-term endurance training results in a muscle-specific decrease of myostatin mRNA content in the rat. Acta physiologica scandinavica. 2005; 183(3):299-307. [DOI:10.1111/j.1365-201X.2005.01406.x] [PMID]

[11] Gumucio JP, Sugg KB, Mendias CL. TGF- $\beta$ superfamily signaling in muscle and tendon adaptation to resistance exercise. Exerc Sport Sci Rev. 2015; 43(2):93-9. [DOI:10.1249/JES.0000000000000041] [PMID] [PMCID]

[12] Jaafari Sardoui S, Nikoei R, Sheibani V. The effect of time series of resistance training on TGF- $\beta 1$ expression and muscle hypertrophy in male wistar rats. J Appl Exerc Physiol. 2015; 11(22):23-32. [DOl:10.22080/ JAEP.2016.1205]

[13] Pfaffl MW. A new mathematical model for relative quantification in real-time RT-PCR. Nucleic Acids Res. 2001; 29(9):e45. [DOI:10.1093/ nar/29.9.e45] [PMID] [PMCID]

[14] Heinemeier K, Olesen J, Haddad F, Langberg H, Kjær M, Baldwin K, et al. Expression of collagen and related growth factors in rat tendon and skeletal muscle in response to specific contraction types. J Physiol. 2007; 582(3):1303-16. [DOI:10.1113/jphysiol.2007.127639] [PMID] [PMCID]

[15] Yang G, Crawford RC, Wang JH. Proliferation and collagen production of human patellar tendon fibroblasts in response to cyclic uniaxial stretching in serum-free conditions. J Biomech. 2004; 37(10):1543-50 [DOI:10.1016/j.jbiomech.2004.01.005] [PMID]

[16] Attarzadeh Hosseini SR, Moeinnia N, Motahari Rad M. The ef fect of two intensities resistance training on muscle growth regulatory myokines in sedentary young women. Obes Medi. 2017; 5:25-8. [DOI:10.1016/j.obmed.2017.01.004]

[17] Matsakas A, Bozzo C, Cacciani N, Caliaro F, Reggiani C, Mascarello $F$, et al. Effect of swimming on myostatin expression in white and red gastrocnemius muscle and in cardiac muscle of rats. Exp Physiol. 2006 91(6):983-94. [DOI:10.1113/expphysiol.2006.033571] [PMID]

[18] Carlson CJ, Booth FW, Gordon SE. Skeletal muscle myostatin mRNA expression is fiber-type specific and increases during hindlimb unloading. Am J Physiol. 1999; 277(2):R601-6. [DOI:10.1152/ ajpregu.1999.277.2.R601] [PMID]

[19] Wehling M, Cai B, Tidball JG. Modulation of myostatin expression during modified muscle use. FASEB J. 2000; 14(1):103-10. [DOI:10.1096/ fasebj.14.1.103] [PMID]

[20] Mendias CL, Lynch EB, Gumucio JP, Flood MD, Rittman DS, Van Pelt DW, et al. Changes in skeletal muscle and tendon structure and function following genetic inactivation of myostatin in rats. J Physiol. 2015 593(8):2037-52. [DOI:10.1113/jphysiol.2014.287144] [PMID] [PMCID] 